\title{
Fatal Stevens-Johnson syndrome following urography with iopamidol in systemic lupus erythematosus
}

\author{
John S. Savill, ${ }^{1}$ Richard Barrie, ${ }^{2}$ Soumitra Ghosh, ${ }^{2}$ Mark Muhlemann, ${ }^{3}$ Peter \\ Dawson $^{4}$ and Charles D. Pusey ${ }^{1}$
}

Departments of ${ }^{1}$ Medicine and ${ }^{4}$ Radiology, Royal Postgraduate Medical School, Hammersmith Hospital, Du Cane Road, London W12 OHS, and the Departments of ${ }^{2}$ Medicine and ${ }^{3}$ Dermatology, Central Middlesex Hospital, Acton Lane, London NW10 7NS, UK.

\begin{abstract}
Summary: A 46 year old white woman with active systemic lupus erythematosus developed a skin rash 8 hours after intravenous urography with the non-ionic contrast medium iopamidol. Severe Stevens-Johnson syndrome with erythema multiforme, intrahepatic cholestasis, pulmonary infiltrates and acute renal failure ensued, leading to her death. Although non-ionic contrast media are generally less toxic than traditional ionic agents, their use in patients with immunological disease may be hazardous.
\end{abstract}

\section{Introduction}

Non-ionic radiological contrast media are generally considered to be safer than conventional agents. ${ }^{1}$ However, fatal vasculitis following urography with iohexol has been reported, in a patient with hydralazine-induced lupus syndrome. ${ }^{2}$ We now report a similar fatality following administration of iopamidol to a patient with active systemic lupus erythematosus (SLE).

\section{Case report}

A 46 year old white housewife presented with a 5week history of malaise, weight loss of $5 \mathrm{~kg}$, and painful swelling of the wrists and small joints of the hands. For 16 years she had suffered recurrent episodes of fever, skin rash and polyarthralgia, with elevation of the erythrocyte sedimentation rate (ESR) and positive antinuclear factor (ANF), but there was no previous evidence of renal disease. Examination of the urine showed +++ protein and $4 \times 10^{7}$ red cells/litre, and renal function had deteriorated with serum creatinine $142 \mu \mathrm{mol} / 1$. ESR was elevated at $80 \mathrm{~mm} / \mathrm{h}$, ANF was positive at a titre of $>1$ in 320 with a diffuse pattern, and serum complement levels were depressed with C3 $57 \%$ normal human serum (normal range $65-110 \%$ ) and C4 $41 \%$ normal human serum (70-110\%).

Correspondence: J.S. Savill M.R.C.P.

Accepted: 4 November 1987
Active SLE with renal involvement was diagnosed and in anticipation of a renal biopsy intravenous urography was performed using $100 \mathrm{~mL}$ of iopamidol $300 \mathrm{mg} / \mathrm{ml}$ ('Niopam 300', E. Merck). a dose appropriate for her weight and renal function. Eight hours later the patient developed a blistering rash involving the limbs and buttocks, with painful ulcers in the mouth and nares. After three days she was admitted to hospital with a pyrexia of $38.8^{\circ} \mathrm{C}$ and large ulceronecrotic plaques, some with bullous changes, on the skin of the extensor aspects of the limbs and trunk (Figure 1). Fresher areas were more typical of the target lesions of erythema multiforme. There was oral ulceration and nasal crusting. General examination

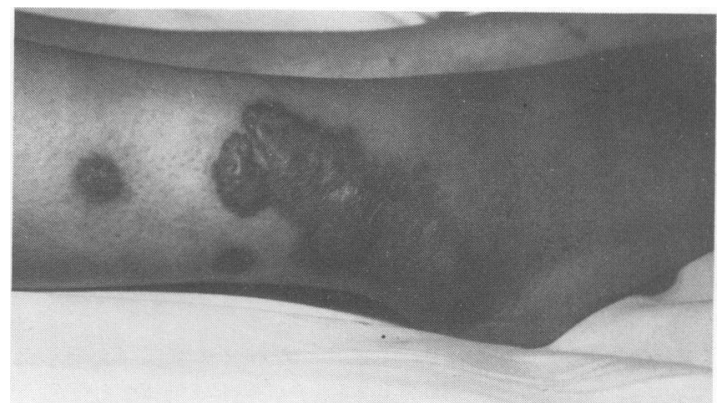

Figure 1 Appearance of skin lesions on lower limbs at presentation.

(C) The Fellowship of Postgraduate Medicine, 1988 
was otherwise normal except for wheezes at the bases, with blood pressure $120 / 70 \mathrm{mmHg}$.

Investigations now showed serum creatinine of $448 \mu \mathrm{mol} / 1$ and examination of the urine revealed ++++ protein with $10^{7}$ white cells and $2 \times 10^{8}$ red cells/l. There was normocytic normochromic anaemia (haemoglobin $10.6 \mathrm{~g} / \mathrm{dl}$ ), white cell count $3.1 \times 10^{9} / 1$ with lymphopaenia on the film, and platelet count $173 \times 10^{9} / 1$. DNA binding was $52 \%$ (upper limit of normal 30\%) and serum complement measurements showed C3 31\% NHS and C4 $18 \%$ NHS. Histopathological examination of biopsies of affected skin showed severe lymphohistiocytic infiltrate throughout the dermis, marked oedema and focal epidermal necrosis with subepidermal bulla formation, compatible with erythema multiforme. Indirect immunofluorescence of involved and non-lesional skin showed linear bands of $\operatorname{IgM}$ and $\mathrm{C} 3$ at the dermo-epidermal junction consistent with the underlying SLE.

A diagnosis of iopamidol-induced StevensJohnson syndrome complicating active lupus nephritis was made, and treatment commenced with high dose dexamethasone. Forty-eight hours after admission she deteriorated with the development of cyanosis, tachypnoea, oliguria and patchy shadowing in the right lower zone on chest radiography. Shortly after transfer to the intensive care unit she suffered a cardio-respiratory arrest from which she was resuscitated, and treatment with artificial ventilation, intravenous fluid replacement, inotropic drugs and a broad spectrum antibiotic (cefotaxime $1 \mathrm{~g}$ twice daily) was started. Bilateral pulmonary infiltrates developed but cleared on the treatment described, and blood and sputum cultures remained negative. Oliguria persisted and serum creatinine rose steadily to $660 \mu \mathrm{mol} / 1$. Pericarditis developed at the end of the first week, and persisted despite the institution of peritoneal dialysis, possibly due to active SLE.

Cyclophosphamide $70 \mathrm{mg}$ /day was added to the steroid therapy, but was stopped one week later because new diffuse alveolar and interstitial infiltrates on chest radiography suggested opportunist fungal infection. Amphotericin was administered until examination of an open lung biopsy had excluded infection. Histology showed intra-alveolar haemorrhage and organising alveolar damage. Jaundice appeared 8 days after admission and despite biochemical evidence of progressive cholestasis repeated ultrasound examinations showed no evidence of extra-hepatic biliary obstruction. Liver biopsy taken 4 weeks after admission revealed toxic necrosis of the bile duct epithelium, with luminal obstruction by inspissated bile, a pattern suggestive of drug toxicity.
Despite full supportive therapy, including dialysis, ventilation and parenteral nutrition, she slowly deteriorated and died 6 weeks after admission. Subsequent renal histology revealed proliferative glomerulonephritis together with acute tubular necrosis.

\section{Discussion}

Administration of iopamidol to this patient with active SLE was associated with the development of a progressive and eventually fatal multi-system illness, the pattern of organ involvement being consistent with Stevens-Johnson syndrome. ${ }^{3,4}$ Neither the manufacturers nor the Committee on Safety of Medicines had been notified of other cases. However, Goodfellow et al. ${ }^{2}$ described a similar illness in a patient with hydralazine-induced lupus given iohexol.

Active SLE probably accounted for many of the clinical features in our case, in particular nephritis, pericarditis and anaemia. The clinical and histological diagnosis of the skin eruption was erythema multiforme; although this lesion has been reported in association with $\mathrm{SLE},{ }^{5}$ the rapid onset of a severe extensive lesion so soon after iopamidol administration suggested a causal relationship. The contrast agent may also have been responsible for progressive cholestasis, since none of the other agents administered are likely to cause this pattern of hepatotoxicity. The cause of the diffuse pulmonary infiltrates developing 2 weeks after admission is less clear, as the histological appearances were compatible with either a drug reaction or lupus pneumonitis. The latter seems less likely however, since the lung lesions became worse despite immunosuppression. Overall, we believe that this clinical picture was due to iopamidol-induced Stevens-Johnson syndrome. ${ }^{4}$

Non-ionic contrast media have attained popularity because of a lower incidence of adverse effects than traditional ionic media. ${ }^{1}$ However, severe toxic reactions have been associated with iohexol $^{2}$ and metrizamide. ${ }^{6}$ In the context of patients with SLE it is of interest that contrast agents, including non-ionic media, have been shown to be capable of in vitro complement activation,? and there is clinical and experimental evidence to suggest that hypocomplementaemia may predispose to contrast reactions. ${ }^{8,9}$ The pathogenetic significance of hypocomplementaemia prior to contrast administration in our patient remains uncertain.

In conclusion, this case and the reports of Goodfellow et $a .^{2}$ and Gelmers ${ }^{6}$ suggest a degree 
of caution in the administration of non-ionic contrast media to patients with SLE. Although it is apparent that the majority of cases do not suffer such adverse effects, the risks of intravenous urography could be avoided in many patients since ultrasonography may provide similar information.

\section{References}

1. Dawson, P., Heron, C. \& Marshall, J. Intravenous urography with low-osmolality contrast agents: theoretical considerations and clinical findings. Clin Radiol 1984, 35: 173-175.

2. Goodfellow, T., Holdstock, G.E., Brunton, F.J. \& Bamforth, J. Fatal acute vasculitis after high-dose urography with iohexol. Br J Radiol 1986, 59: 620-621.

3. Claxton, R.C. A review of 31 cases of Stevens-Johnson syndrome. Med J Aust 1963, i: 963-967.

4. Bianchine, J.R., Macareg, P.V.J., Lasagna, L. et al. Drugs as etiologic factors in the Stevens-Johnson syndrome. Am J Med 1968, 44: 390-405.

5. Rowell, N.R., Beck, J.S. \& Anderson, J.R. Lupus erythematosus and erythema multiforme-like lesions. Arch Dermatol 1963, 88: 176-180.

\section{Acknowledgements}

We acknowledge the medical, nursing and technical staff involved in the care of this patient, and are grateful to Miss S. Lydon for preparing the manuscript. JSS is a Medical Research Council Training Fellow and CDP is a Wellcome Senior Research Fellow in Clinical Science.
6. Gelmers, H.J. Exacerbation of systemic lupus erythematosus, aseptic meningitis and acute mental symptoms following metrizamide lumbar myelography. Neuroradiology 1984, 26: 65-66.

7. Dawson, P., Turner, M.W., Bradshaw, A. \& Westaby, $\mathrm{S}$. Complement activation and generation of $\mathrm{C} 3 \mathrm{a}$ anaphylotoxin by radiological contrast agents. $\mathrm{Br} \mathrm{J}$ Radiol 1983, 56, 447-448.

8. Lasser, E.C., Slovak, M. \& Lang, J.H. Development of contrast media idiosyncrasy in the dog. Radiology 1976, 119: 91-95.

9. Lasser, E.C., Lang, J.H., Lyon, S.G. \& Hamblin, A.E. Complement and contrast material reactions. $J$ Allergy Clin Immunol 1979, 64: 105-112. 